\section{PTU-003 CIRCULATING LEVELS OF INTERLEUKIN-18 CORRELATE WITH SEVERITY FOLLOWING HUMAN ACUTE LIVER INJURY}

doi:10.1136/gutjnl-2012-302514c.3

${ }^{1} \mathrm{D}$ G Craig, ${ }^{*}{ }^{2} \mathrm{~A}$ Conway-Morris, ${ }^{3} \mathrm{P}$ Lee, ${ }^{3} \mathrm{~A}$ Pryde, ${ }^{2} \mathrm{~J}$ Simpson, ${ }^{3} \mathrm{P} C$ Hayes, ${ }^{3} \mathrm{~K}$ J Simpson. ${ }^{1}$ Scottish Liver Transplantation Unit, Royal Infirmary of Edinburgh, Edinburgh, UK; ${ }^{2}$ MRC Centre for Inflammation Research, University of Edinburgh, Edinburgh, UK; ${ }^{3}$ Division of Clinical and Surgical Sciences, University of Edinburgh, Edinburgh, UK

Introduction Excessive innate immune activation may precipitate multiorgan failure following paracetamol overdose (POD). Identification of amplification loops in this process could reveal novel therapeutic targets. Interleukin (IL)-18 is a potent proinflammatory cytokine which stimulates downstream $\mathrm{T}$ helper-1 cell responses and may trigger loss of regulatory natural killer (NK) cells.

Methods Consecutive patients ( $\mathrm{n}=46$, (19 (41.3\%) male) admitted to the Royal Infirmary of Edinburgh with paracetamol-induced acute liver injury (ALT>1000 IU/1 and coagulopathy) were enrolled. IL-18 levels were measured by ELISA. Immunophenotypic analysis of circulating lymphocytes was determined in whole blood by fluorescence-activated cell sorter (FACS) analysis.

Results A total of 29/46 (63.0\%) PODs developed hepatic encephalopathy $(\mathrm{HE})$, and therefore acute liver failure. IL-18 levels were significantly higher in PODs (median 457 (IOR 340-671) pg/mL, $\mathrm{n}=46)$ compared with chronic liver disease $(292(192-591) \mathrm{pg} / \mathrm{ml}$, $\mathrm{n}=15, \mathrm{p}<0.05)$ and healthy $(163(90-191) \mathrm{pg} / \mathrm{ml}, \mathrm{n}=13, \mathrm{p}<0.001)$ controls. Admission IL-18 levels in PODs correlated with both proand anti-inflammatory cytokines such as IL-6 (Spearman's $\mathrm{r}=0.491$, $\mathrm{p}=0.001)$ and IL-10 ( $\mathrm{r}=0.360, \mathrm{p}=0.019)$, with markers of T-cell (IL2$\mathrm{sR} \alpha, \mathrm{r}=0.567, \mathrm{p}<0.0001$ ) and macrophage (neopterin, $\mathrm{r}=0.422$, $\mathrm{p}=0.015$ ) activation, and with organ failure scores (SOFA, $\mathrm{r}=0.485$, $p=0.0007$; APACHE II, $r=0.466, p=0.001$ ). Admission IL-18 levels were significantly higher in PODs who developed HE $(p=0.0006)$ or the systemic inflammatory response syndrome $(p=0.038)$, and in PODs who died/required emergency liver transplantation (OLT, $\mathrm{p}=0.020$; AUC $71.4 \%$ (95\% CI $55.4 \%$ to $87.4 \%$ ). Flow cytometry analysis of peripheral blood lymphocytes revealed a significant decrease in the proportion of CD3-/CD56+ NK cells, with rapid recovery following OLT.

Conclusion IL-18 is associated with innate immune activation and adverse outcomes following POD. Future animal studies should explore IL-18 and NK cell modulation following POD.

Competing interests None declared.

\section{PTU-004 EXTREME HYPERFERRITINAEMIA FOLLOWING PARACETAMOL-INDUCED HUMAN ACUTE LIVER INJURY}

\section{doi:10.1136/gutjnl-2012-302514c.4}

${ }^{1} \mathrm{D}$ G Craig, ${ }^{*} \mathrm{~A}$ Pryde, ${ }^{3} \mathrm{~A}$ Conway-Morris, ${ }^{3} \mathrm{~J}$ Simpson, ${ }^{4} \mathrm{~S}$ W Walker, ${ }^{4} \mathrm{G}$ J Beckett, ${ }^{5} \mathrm{C} O$ Bellamy, ${ }^{2} \mathrm{P}$ C Hayes, ${ }^{2} \mathrm{~K}$ J Simpson. ${ }^{1}$ Scottish Liver Transplantation Unit, Royal Infirmary of Edinburgh, Edinburgh, UK; ${ }^{2}$ Division of Clinical and Surgical Sciences, University of Edinburgh, Edinburgh, UK; ${ }^{3} \mathrm{MRC}$ Centre for Inflammation Research, University of Edinburgh, Edinburgh, UK; ${ }^{4}$ Department of Clinical Biochemistry, Royal Infirmary of Edinburgh, Edinburgh, UK; ${ }^{5}$ Department of Pathology, Royal Infirmary of Edinburgh, Edinburgh, UK

Introduction Activated macrophages may play a critical role in the pathogenesis of acute liver failure (ALF). Serum ferritin (SF) is a circulating marker of macrophage activation, and heavy $(\mathrm{H})$ isoforms of ferritin may have immunostimulatory effects. However, ferritin may be released from necrotic liver, confounding SF interpretation in ALF. The SF/ALT ratio may have prognostic value in non-paracetamol ALF, but has not been examined in patients with paracetamol (POD)-ALF.
Methods Analysis of SF levels in acute liver injury patients admitted to a tertiary liver center, with western blotting and immunohistochemistry for ferritin isoforms.

Results Retrospective database analysis revealed elevated admission SF $(>300 \mu \mathrm{g} / \mathrm{l})$ in $109 / 124(87.9 \%)$ of acute liver injury patients. Extreme SF elevations $(>10000 \mu \mathrm{g} / \mathrm{l})$ were more common in POD $(36 / 71,50.7 \%)$ compared with non-POD patients $(5 / 53,9.4 \%$, $\mathrm{p}<0.001)$. Extremely elevated admission SF was confirmed in a prospective cohort of $47 \mathrm{POD}$ cases (22/47, 46.8\%). In both POD cohorts, admission SF was significantly higher in patients who died/ were transplanted compared with spontaneous survivors $(p=0.001$, AUC 0.722 (95\% CI 0.614 to 0.831 ) and in patients who developed hepatic encephalopathy $(p=0.038$ ) or the systemic inflammatory response syndrome (SIRS, $\mathrm{p}=0.008$ ). Hyperferritinaemia correlated with proinflammatory (IL-6, Spearman's $r=0.442, p=0.006$; IL-8, $\mathrm{r}=0.502, \mathrm{p}=0.001)$ and antiinflammatory (IL-10; $\mathrm{r}=0.349, \mathrm{p}=0.030$ ) cytokine release following $\mathrm{POD}$, and with organ dysfunction (SOFA; $r=0.529, p<0.001)$, but not with serum ALT $(r=0.113$, $\mathrm{p}=0.227)$. The ferritin/ALT ratio did not improve prognostic accuracy in PODs (AUC 0.706 (95\% CI 0.595 to 0.817). Immunohistochemistry confirmed $\mathrm{H}$ and light (L) ferritin isoform expression in both normal liver tissue and explanted tissue from ALF patients. Immunoblotting of serum from POD patients with elevated SF revealed significant amounts of circulating $\mathrm{H}$-ferritin, with no circulating $\mathrm{H}$-ferritin observed in healthy controls.

Conclusion Extreme elevations of SF are common following POD, and are associated with adverse outcomes. SF is a widely available biomarker that may have prognostic value in patients with PODALF and merits further evaluation in larger, prospective studies. The correlation with SIRS, organ failure and cytokinaemia and the observation of circulating $\mathrm{H}$ ferritin also suggests that SF may be a mediator of adverse outcome.

Competing interests None declared.

\section{PTU-005 CIRCULATING LEVELS OF NEOPTERIN ARE ASSOCIATED WITH ADVERSE OUTCOMES FOLLOWING PARACETAMOL- INDUCED ACUTE LIVER INJURY}

doi:10.1136/gutjpl-2012-302514c.5

${ }^{1} \mathrm{D}$ G Craig, ${ }^{*}{ }^{2} \mathrm{P}$ Lee, ${ }^{2} \mathrm{~A}$ Pryde, ${ }^{2} \mathrm{P} \mathrm{C}$ Hayes, ${ }^{2} \mathrm{~K}$ J Simpson. ${ }^{1}$ Scottish Liver Transplantation Unit, Royal Infirmary of Edinburgh, Edinburgh, UK; ${ }^{2}$ Division of Clinical and Surgical Sciences, University of Edinburgh, Edinburgh, UK

Introduction Macrophage activation is implicated in the pathogenesis of multiorgan failure following paracetamol overdose (POD). Simple biomarkers of macrophage activation could aid earlier identification of high-risk POD patients. Neopterin is synthesised from macrophages and monocytes upon stimulation by interferon- $\gamma$ and serum levels reflect the intensity of monocyte/macrophage activation.

Methods Consecutive patients ( $\mathrm{n}=33$, (15 (45.5\%) male) admitted to the Royal Infirmary of Edinburgh with paracetamol-induced acute liver injury (ALT>1000 IU/1 and coagulopathy) were enrolled. Serum neopterin levels were measured by ELISA (IBL International, Hamburg, Germany).

Results A total of 24/33 (72.7\%) PODs developed hepatic encephalopathy $(\mathrm{HE})$, and therefore acute liver failure. Neopterin levels were significantly higher in PODs (median 66.0 (IOR 25.4-96.6) nmol/l) compared with both chronic liver disease (10.8 $(6.7-12.1) \mathrm{nmol} / \mathrm{l}, \mathrm{n}=7, \quad \mathrm{p}<0.001)$ and healthy (11.4 (9.4-15.7) $\mathrm{nmol} / \mathrm{l}, \mathrm{n}=10, \mathrm{p}<0.001)$ controls, but were similar to non-POD acute liver injury patients $(52.5(42.0-113.8) \mathrm{nmol} / \mathrm{l}, \mathrm{n}=8$, $\mathrm{p}>0.05)$. Admission neopterin levels were significantly higher in PODs who developed HE (HE, 72.9 (59.5-116.7) nmol/l, n=24; no $\mathrm{HE}, 20.7(17.5-22.1) \mathrm{nmol} / \mathrm{l}, \mathrm{n}=9, \mathrm{p}<0.0001)$ or the systemic inflammatory response syndrome (SIRS, 79.1 (66.7-116.7) nmol/l, 
$\mathrm{n}=20$; no SIRS, $21.6(18.6-43.1) \mathrm{nmol} / \mathrm{l}, \mathrm{n}=13, \mathrm{p}<0.0001)$, and in PODs who died/required emergency liver transplantation (OLT 72.9 (68.1-119.2) nmol/1, $\mathrm{n}=14$; survived 30.3 (20.7-89.1) $\mathrm{nmol} / \mathrm{l}, \mathrm{n}=29$, $\mathrm{p}=0.006$; AUROC $78.6 \%$ (95\% CI $62.2 \%$ to $94.9 \%$ ). An admission neopterin value of $50 \mathrm{nmol} / \mathrm{l}$ predicted death/OLT with a sensitivity of 100.0 (95\% CI 76.8 to 100.0) and specificity of 63.2 (95\% CI 38.4 to 83.7). Admission neopterin levels in PODs correlated with: serum creatinine (Spearman's $\mathrm{r}=0.847, \mathrm{p}<0.0001$ ); prothrombin time $(\mathrm{r}=0.439, \mathrm{p}=0.011)$; platelet count $(\mathrm{r}=-0.463, \mathrm{p}=0.007)$; IL-6 $(\mathrm{r}=0.650, \mathrm{p}=0.0001) ; \mathrm{IL}-10 \quad(\mathrm{r}=0.529, \mathrm{p}=0.004)$; serum ferritin $(\mathrm{r}=0.467, \mathrm{p}=0.006)$; and with organ failure scores (SOFA, $r=0.725$, $\mathrm{p}<0.0001$; APACHE II, $\mathrm{r}=0.659, \mathrm{p}<0.0001)$.

Conclusion Serum neopterin levels are significantly elevated following POD and are correlated with adverse outcomes. Serum neopterin may have value as an early marker of prognosis in ALF and should be assessed further with larger prospective cohorts of ALF patients. The correlation of serum neopterin with adverse outcomes provides further support for the importance of macrophage activation in the pathogenesis of multiorgan failure in ALF.

Competing interests None declared.

\section{PTU-006 THE SEQUENTIAL ORGAN FAILURE ASSESSMENT (SOFA) SCORE IS AN EFFECTIVE TRIAGE MARKER FOLLOWING STAGGERED PARACETAMOL (ACETAMINOPHEN) OVERDOSE}

doi:10.1136/gutjnl-2012-302514c.6

${ }^{1} \mathrm{D}$ G Craig, ${ }^{*} \mathrm{~S}$ Zafar, ${ }^{2} \mathrm{~T}$ Reid, ${ }^{1} \mathrm{~J}$ Davidson, ${ }^{1} \mathrm{~K}$ Martin, ${ }^{3} \mathrm{P} \mathrm{C}$ Hayes, ${ }^{3} \mathrm{~K} \mathrm{~J}$ Simpson. ${ }^{1}$ Scottish Liver Transplantation Unit, Royal Infirmary of Edinburgh, Edinburgh, UK; ${ }^{2}$ University of Edinburgh, Edinburgh, UK; ${ }^{3}$ Division of Clinical and Surgical Sciences, University of Edinburgh, Edinburgh, UK

Introduction The Sequential Organ Failure Assessment (SOFA) score is an effective triage marker following single time point paracetamol (acetaminophen) overdose, ${ }^{1}$ but has not been evaluated following staggered paracetamol overdose. The aims of this study were to evaluate the prognostic accuracy of the SOFA score in a cohort of severe acute liver injury patients following staggered paracetamol overdose.

Methods Time-course analysis of 50 staggered paracetamol overdoses admitted to a tertiary liver centre. Individual laboratory samples were correlated with the corresponding clinical parameters in relation to time from admission, and the daily SOFA score calculated.

Results A total of $39 / 50$ (78\%) patients developed hepatic encephalopathy, and therefore acute liver failure. The area under the SOFA receiver operator characteristic for death/liver transplantation was 87.4 (95\% CI 73.2 to 95.7), 94.3 (95\% CI 82.5 to 99.1), and 98.4 $(95 \%$ CI 84.3 to 100.0$)$ at 0,24 , and $48 \mathrm{~h}$ respectively post-admission. A SOFA score of $<6$ at tertiary care admission predicted survival with a sensitivity of $100.0 \%(95 \%$ CI $76.8 \%$ to $100.0 \%)$ and specificity of $58.3 \%$ (95\% CI $40.8 \%$ to $74.5 \%$ ), compared with $85.7 \%$ ( $95 \%$ CI $60.6 \%$ to $97.4 \%$ ) and $75.0 \%$ (95\% CI $65.2 \%$ to $79.5 \%)$ respectively for the modified Kings College criteria. Only $2 / 21$ patients with an admission SOFA score $<6$ required renal replacement therapy or intracerebral pressure monitoring. SOFA significantly outperformed the Model for End-stage Liver Disease at 0 $(p=0.0013), 24 \quad(p=0.0001)$ and $48 \mathrm{~h} \quad(p=0.0193) \quad$ following admission.

Conclusion A SOFA score $<6$ at tertiary care admission following a staggered paracetamol overdose carries a high negative predictive value. The SOFA score could improve triage of high risk staggered paracetamol overdose patients.

\section{Competing interests None declared.}

\section{REFERENCE}

1. Craig DG, Reid TW, Martin KG, et al. The systemic inflammatory response syndrome and sequential organ failure assessment scores are effective triage markers following paracetamol (acetaminophen) overdose. Aliment Pharmacol Ther 2011;34:219-28.

\section{PTU-007 CEREBRAL OEDEMA IS RARE IN ACUTE-ON-CHRONIC LIVER FAILURE}

doi:10.1136/gutjnl-2012-302514c.7

${ }^{1} \mathrm{D}$ Joshi, ${ }^{1} \mathrm{~A}$ Patel, ${ }^{1} \mathrm{~J}$ O'Grady, ${ }^{1} \mathrm{D}$ Shawcross, ${ }^{2} \mathrm{~S}$ Connors, ${ }^{1} \mathrm{C}$ Willars, ${ }^{1} \mathrm{~W}$ Bernal, ${ }^{1} \mathrm{~J}$ Wendon, ${ }^{1} \mathrm{G}$ Auzinger. ${ }^{1}$ Institute of Liver Studies, King's College Hospital, London, UK; '2Department of Neuro-radiology, King's College Hospital, London, UK

Introduction Acute-on-chronic liver failure (AoCLF) has a rapidly progressive disease course associated with significant mortality. Hepatic encephalopathy (HE) is common associated with hyperammonemia, systemic inflammation and hyponatremia. The prevalence of cerebral oedema in AoCLF is unknown. We aimed to describe the prevalence of cerebral oedema in a cohort of AoCLF adult ( $>18$ years) patients admitted to the liver intensive treatment unit (LITU) between January 2005 and 2011.

Methods AoCLF was defined using criteria of Sarin et al. ${ }^{1}$ Arterial ammonia (NH3), MELD, UKELD, and organ failure (SOFA) scores were collated (results expressed as medians with ranges). Patients who had undergone cranial CT imaging were identified. Neuroimages were reported by consultant neuro-radiologists.

Results During the study period, 1008 patients with chronic liver disease (CLD) were admitted to the LITU. 173 patients (110 male) underwent neuro-imaging. Of these 81 (48 male) fulfilled criteria for AoCLF, Over the same time period 655 patients were admitted with acute liver failure. Variceal bleeding (30\%) and sepsis (31\%) were the most frequent precipitants of AoCLF. Compared to the CLD group, AoCLF patients were younger $(50,24-71$ vs $59,30-74, p=0.001)$, serum NH3 $(143,40-305$ vs $111,28-315)$, grade of $H E(3,1-4$ vs 1 , $0-4)$, MELD (25, 8-40 vs $15,6-34)$, SOFA (11, 2-17 vs 4, 0-14), UKELD (63, $50-75$ vs $55,44-73)$ and SIRS score $(2,1-3$ vs $1,0-3)$ were higher $(p<0.0001$ for all). Serum sodium was lower in the AoCLF group (132 118-154 vs 136120-146, $p<0.0001)$. HE ( $\geq$ grade 3 ) occurred in $66 \%$ of AoCLF patients vs $13 \%$ CLD ( $p<0.0001)$. In those with neuro-imaging, $26 \%$ were normal, $26 \%$ demonstrated increased cerebral atrophy for age, $15 \%$ small vessel disease and $10 \%$ intra-cranial haemorrhage. Cerebral oedema was seen in two patients with AoCLF, 1 post TIPSS (NH3 $289 \mu \mathrm{mol} / \mathrm{l}$ ) and 1 with septic shock (NH3 $268 \mu \mathrm{mol} / \mathrm{l}$ ). 72 patients with ALF underwent neuro-imaging with $32 \%$ showing radiological evidence of cerebral oedema. Compared to the CLD group, 30 and 90 day survival was poorer in AoCLF $(52 \%$ and $42 \%$ vs $80 \%$ and $75 \%$, log rank $\mathrm{p}<0.0001)$. The mode of death was that of progressive multi-organ failure (MOF). The two patients with cerebral oedema on CT suffered cerebral deaths with tonsillar herniation. AUROC analysis for survival of AoCLF identified SOFA $(0.67,95 \%$ CI 0.54 to 0.8 , $\mathrm{p}=0.02)$ MELD $(0.74,95 \%$ CI 0.61 to $0.87, \mathrm{p}<0.0001)$ and UKELD (0.87, 95\% CI 0.8 to $0.97, \mathrm{p}<0.0001)$.

Conclusion Our data demonstrates poor outcome in patients with AoCLF compared to those with CLD requiring admission to LITU. Mortality was attributable to MOF and although deep levels of encephalopathy requiring ventilation were common (66\%), the prevalence of cerebral oedema was rare at $2 \%$.

Competing interests None declared.

\section{REFERENCE}

1. Sarin, et al. Hep Int 2009. 